\title{
A Decision Frameworkon Small and Medium Enterprises to Adopt Cloud Software as a Service
}

\author{
Hsi-Peng Lu ${ }^{1}$ and Yao-Yuan Liu ${ }^{2, a}$ \\ ${ }^{1}$ Department of Information Management, National Taiwan University of Science and Technology, Taipei, Taiwan, ROC. \\ ${ }^{2}$ Graduate school of Management, National Taiwan University of Science and Technology, Taipei, Taiwan, ROC
}

\begin{abstract}
Software as a service (SaaS)is the future trend in software services. However, only a few successful SaaS providerscurrently exist. This study investigated how Taiwanese small and medium enterprises (SMEs) can utilize SaaS, giveSaaSprovidersbusiness strategy references, and supply a basis for information system operators to transfer service mode in order to innovate the provision of SaaS. The study is based on survey results obtained from 474 SMEs for a telecom company in Taiwan to determine the reasons SMEs utilizeSaaS. Taking the technology acceptance model (TAM) as the fundamental theoretical foundation, we explored the perceived usefulness, perceived ease of use, and SaaS system features as external variables. In addition, we analyzed each system characteristic on the Likert scale and found that, in addition to the necessary consideration for cost and convenience, SaaS also provides information and communication technology applications in system characteristics, which are including online sharing services, customer relationship management services, and business operation and orders purchasing services. The fact that SMEs wish to utilize SaaS has a positive and direct impact on business.
\end{abstract}

\section{Introduction}

\subsection{Research rationale}

Traditionally, small and medium enterprises (SMEs)have utilized software packages to manage information because their resources are limited, and they cannot afford the high cost involved in the development of information systems. However, the data contained in software packages are prone to degradation, which leadsto situations in which risk estimation and operational growth are compromised. Software as a service (SaaS) has cross-platform, cross-network technical capabilities that are not subject to time and space constraints, and is consequently flexible, stable, scalable, and price efficient. Depending on the specific usage desiredby SMEs, SaaS can be utilized anytime and anywhere, via mobile technology on emerging broadband and $4 \mathrm{G}$ networks. SMEs can thus expand their future operation efficiency and strengthen their competitive advantage by utilizing SaaS.

\subsection{Purpose}

Previous studies conducted on SaaS focused on the reasons why SMEs are using cloud services and determined that SMEs benefit from the framework and effectiveness of improved enterprise terminal IT services, as well as improved cost benefits, information security, and network privacy and stability. However, the relationship between

SaaS system characteristics and their use by SMEs has not been adequately investigated. In this study, we employed the technology acceptance model (TAM)and exploredthe factors and variables that affect the behavior and intention of SMEs that use cloud services (in particular, SaaS). The results of this study will supply SaaS providers with a reference for business strategy and will help IT operators to develop SaaS that conform to the needs of SMEs and facilitate innovation in the provision of SaaS.

\section{Literaturereview}

\subsection{Software as a service (SaaS)}

Cloud resources can be dynamically extended and configured and applications modularizedto accord withenterpriseneeds. Further, SMEs can purchase authorization from suppliers to use services such as office, back-enddataanalysis,customerrelationship management, business process management, and human resources management applications according to their own needs. Users can experience software and services simply by connecting to the Internet, thereby eliminating information systems construction and software licensing costs. Further, resources can be distributed in accordance 
with the needs of users and payment remitted according to actual usage. Examples of such software services include Amazon's AWS, Salesforce's CRM, andMicrosoft's Office 365.

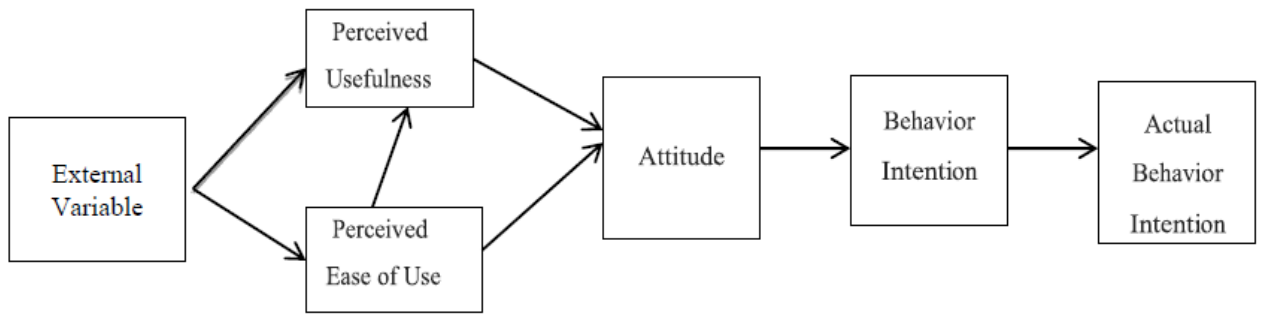

Figure 1. Technology acceptance model (Davis, 1986) [1]

\subsection{The technology acceptance model (TAM)}

The technology acceptance model (TAM) as shown in Figure 1 was proposed byDavis [1]. It was developed based on rational action theory [2], self-efficiency, the cost-benefit model, and innovation. Similar to rational action theory, TAM holds that belief affects attitude, attitude further influences behavioral intention, and behavioral intention is the best variable with which to predict behavior [3]. Behavioral intention refers to the subjective probability of a person performing certain actions, theorizing that the stronger the intention, the higher the likelihood that such actions are actually performed [4]. TAM is employed primarily to explain the crucial factors of users accepting science and technology systems. Davis et al.[5] abandoned the subjective norm in TRA by means of experimentation, explaining that the acceptance intention for users of a specific system is affected by perceptions and beliefs, such as perceived usefulness and perceived ease of use. TAM, by definition, measures usefulness as a result of performance improvement users expect in particular application systems, and perceives ease of use as a result of expected effort dispensation by users.
TAM classifies system features, users' characteristics, organization factors, and training as external variables that influence acceptance and use by users. However, the effect is relatively small compared to that of perceived usefulness and perceived ease of use,andTAM does not indicate which variables affect perceived usefulness and perceived ease of use. TAM has been verified and is highly recognized. It is applied in various fields, including technology products and services, and in the continued development of TAM [6]. The core architecture of TAM is highly reliable and can be introduced in all categories [7]. From the theory and empirical results of TAM, it can be seen that the actual usage will be affected by behavior intention, and the behavior intention is further influenced by the user's attitude and cognizance. Furthermore, user attitude is decided by the cognition of usefulness and ease of use. Venkatesh and Bala[8] summarized the research conducted on TAM, and found that they focused on three areas: psychological viewpoint focusing on TAM architecture, verification and support of TAM structure, and increasing facets to expand TAM architecture. Subsequently, they developed a representative theoretical framework indicating four different determinants (i.e., external variables, which affect the perceived usefulness and perceived ease of use): individual differences, system characteristics, social influence and facilitating conditions (Figure 2).

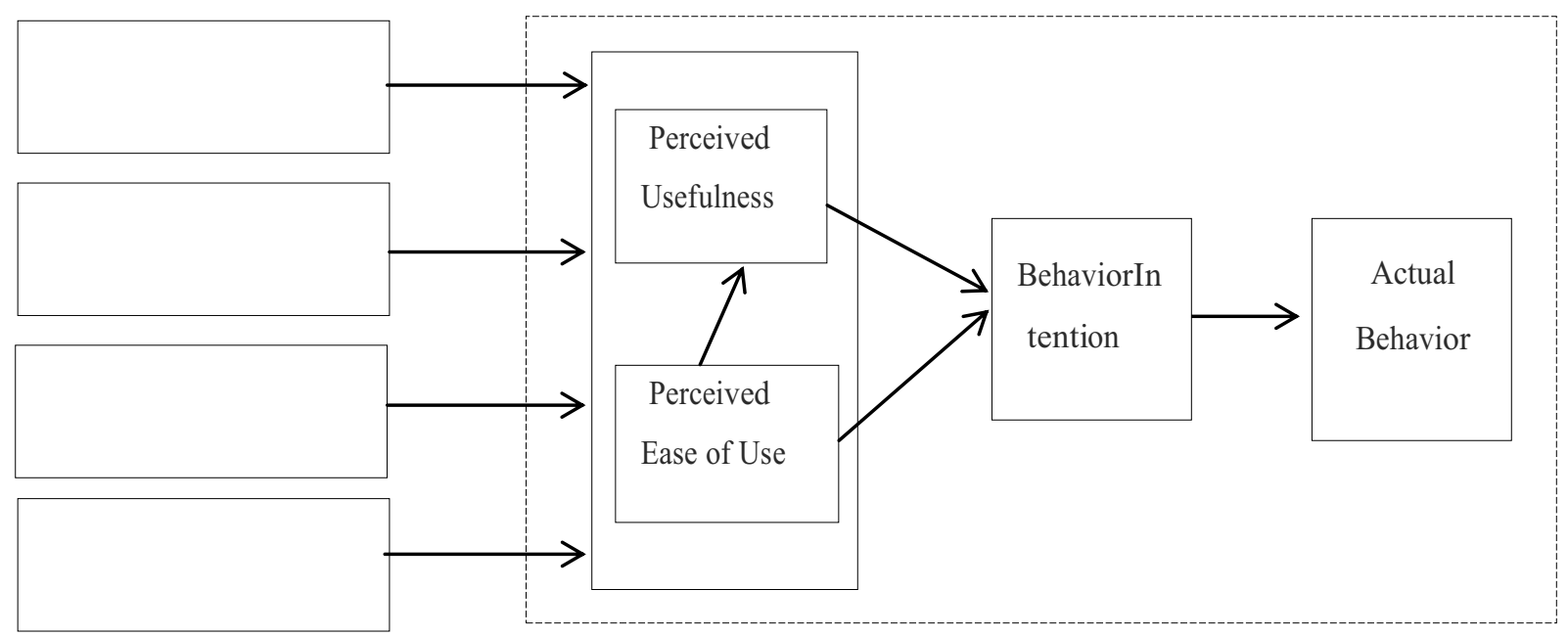

Figure 2. Theoretical architecture: determinants of perceived usefulness and perceived ease of use (Venkatesh andBala, 2008)[8]. 


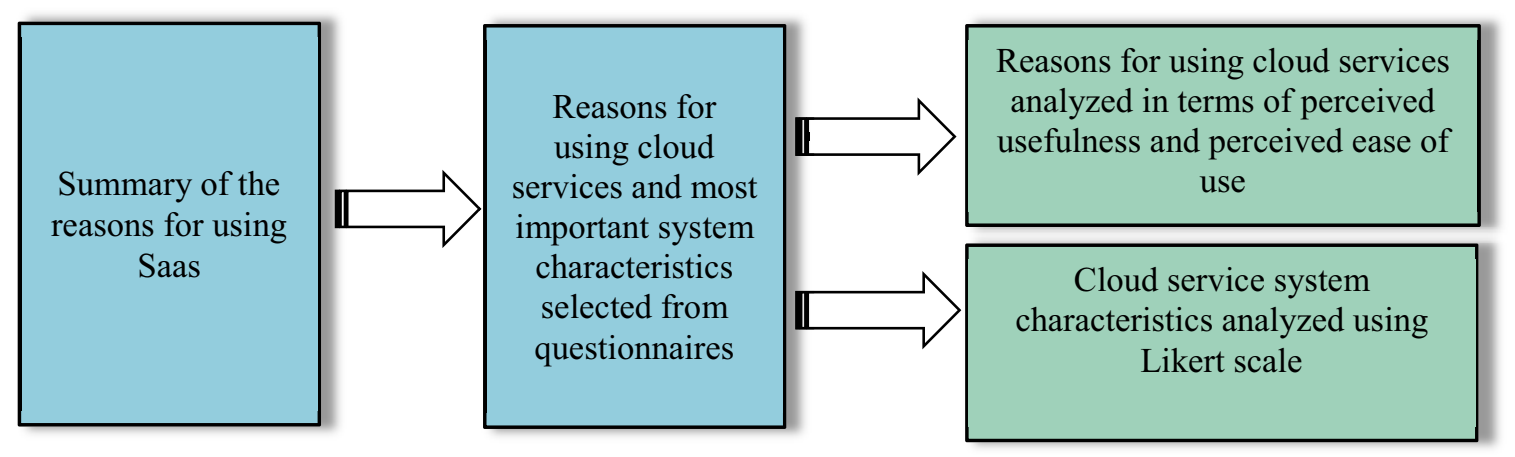

Figure 3. Rationale for using cloud service and system characteristics analysis procedure

\subsection{Extension of TAM}

TAM is widely applied in qualitative research, and many scholars have expanded TAM to improve the interpretations of user acceptance behaviors. King and $\mathrm{He}$ [9] reviewed 88 publications and proposed a more general architecture based on TAM. They divided research on TAM into four categories: (1) Research on external variables, including situation involvement, prior use or experience [10] and computer self-efficacy [11]; (2) improvement of the prediction of TAM for behavior intention by combining with other theories, including subjective standard and expectation [12], task-science and technology [13], and risk and trust [14]; (3) situational factors with moderating effect, such asscience and technology characteristics; and (4) subsequent measurements, such as attitude, perceived use, and actual use[15].

\section{Research design}

\subsection{Research method}

The study consisted of survey questionnaires, and the responses from Taiwanese companies were used to determine the main factors to consider when importing cloud application systems for such users. A flowchart of the research method is presented in Figure 3.

\subsection{Establishment of research model and hypothesis}

\subsubsection{Survey design}

The survey questionnairestargeted technology managers. The contents of the questionnaires were divided into three sections: basic information for enterprises, reasons for using cloud services, and, most importantly, cloud system characteristics. Thus, the first category(the enterprise category) dealt with number of computers and number of employees. The second category was the main consideration for using SaaS. The third category was concerned with the most crucial characteristics of SaaS The main purpose of the questionnaireswas to determine the reasons for using SaaS and the characteristics of SaaS systems.

This study found that enterprises use cloud services for 12 main reasons (reduce build and maintenance costs, pay fees according to the usage, improve employee communication efficiency, attempt to apply technology in response to trends, can be used anywhere, centrally manage data, fast and easy to use, can be used in mobile terminal, high system expansion elasticity, access to the latest applications and services, fast computing speed, and high security) collected from various journals, publications, books, and vendor information, and seven service system characteristics (information and communication application and online sharing service, office administration service, CRM services, operation order management service, operation financial management service, human resource management service, business operation and orders production and manufacturing management services).These contents were all designed into the questionnaire.

\subsubsection{Sampling method and survey object}

The survey targeted 474 SMEs. Questionnaires were mailed to information managers in the SMEs, who were requested to respond.

\subsection{The information analysis method}

In this study, perceived usefulness and perceived ease of use were analyzed on a simple scale, and the system characteristics analyzed using the Likert-type total formula scale. The subjects were asked to respond usinga specific score to reflect the extent of their favor, which can help to identify the major factors in cloud computing services that enterprises consider among the system characteristics. In order to conform to the Likert construction and testing rules, the subjects were required to select one offive favor grades. Numberswere assigned to the values of the individual options, with two representing the least agreeable, and 10 the most agreeable. 


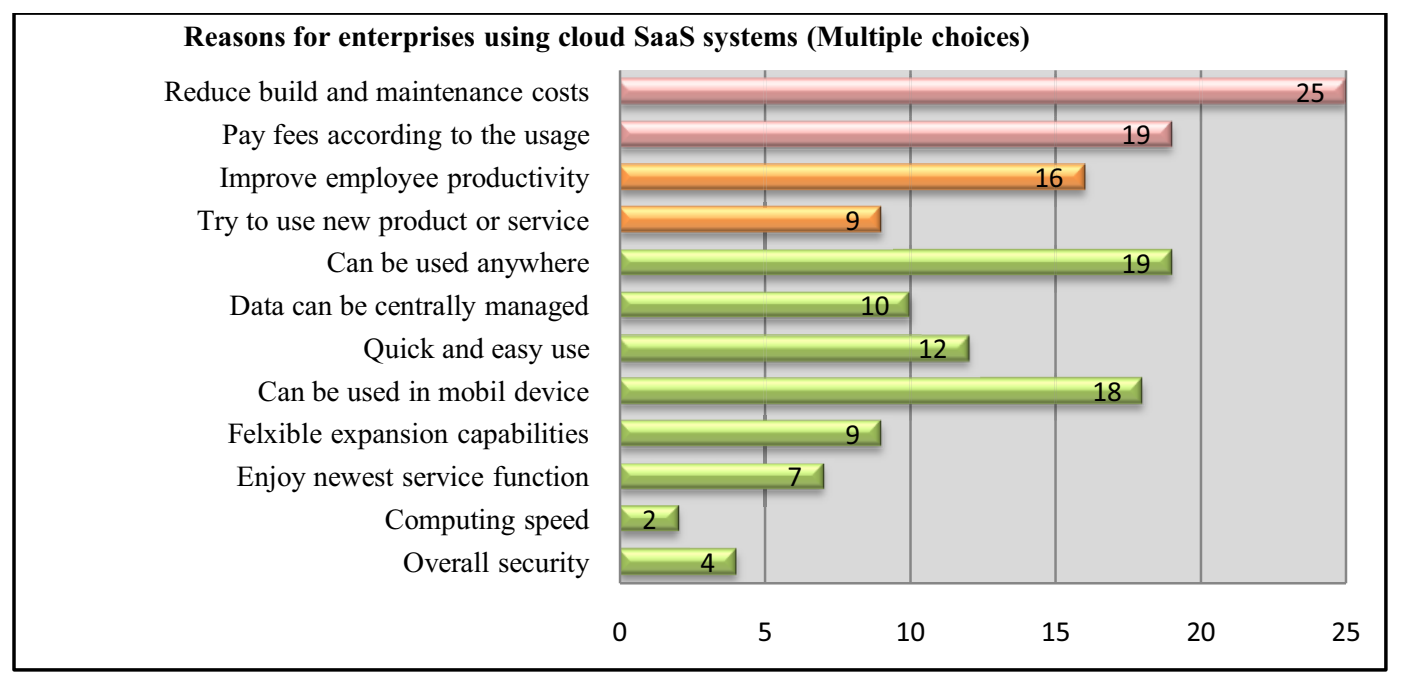

Figure 4. Questionnaire statistics showing crucial reasons enterprises use cloud applications.

\section{Analysis of results}

\subsection{Analysis of basic enterprise information}

In order to understand the sample structure of the returnedquestionnaires, the basic information pertaining to the objectives in the study was analyzed using the descriptive statistical method. Statistical variables of research objectives included industry category, the number of employees (Table 1), and the number of computers (Table 2). In terms of industry category responses, the majority sampled were information service corporations, with 19 units in total accounting for $35.8 \%$, followed by members of the electric and electronic machinery industry, with 11 units accounting for $20.8 \%$.

\subsection{Survey results and analysis}

In this study, 474 questionnaires were sent by e-mail, and 53 responses were received - a return rate of $11.2 \%$. From the response results, we found that among enterprises that had the intention of using commercial operation application services, the top five factors for employing SaaS were reduced build and maintenance costs, pay fees according to the usage structure, improved employee work efficiency, can be used anywhere, and mobile device applications (Figure 4) according to the summarization and categorization of perceived usefulness, perceived ease of use, and external variables (Table 3). The main consideration as to whether to purchase a cloud application was cost. Convenience was also an important determinant, followed by the function of system applications, by which they expect to improve employee work efficiency. In terms of system applications, analyzing demand strength on the Likert scale, the enterprises using commercial operation services are in maximum demand of ICT applicationsand online sharing services, followed by business orders and purchasing operation services, and CRM services (Table 4).
Table 1.Quantitative statistics for the number of employees in corporations that replied to the questionnaire.

\begin{tabular}{|c|c|c|c|}
\hline \multirow{4}{*}{ Item } & Category & $\begin{array}{c}\text { Number of } \\
\text { returned } \\
\text { questionnaires }\end{array}$ & Proportion \\
\hline \multirow{4}{*}{$\begin{array}{c}\text { Number } \\
\text { of } \\
\text { employees }\end{array}$} & $1-5$ & 11 & $20.7 \%$ \\
\cline { 2 - 4 } & $6-10$ & 5 & $9.4 \%$ \\
\cline { 2 - 4 } & $11-20$ & 6 & $11.3 \%$ \\
\cline { 2 - 4 } & $21-50$ & 9 & $16.9 \%$ \\
\cline { 2 - 4 } & $101-200$ & 7 & $16.9 \%$ \\
\cline { 2 - 4 } & $201-500$ & 2 & $13.2 \%$ \\
\cline { 2 - 4 } & Over 501 & 4 & $7.5 \%$ \\
\hline
\end{tabular}

Table 2.Quantitative statistics for the number of computers in corporations that replied to the questionnaire.

\begin{tabular}{|c|c|c|c|}
\hline \multirow{4}{*}{ Item } & Category & $\begin{array}{c}\text { Number of } \\
\text { returned } \\
\text { questionnaires }\end{array}$ & Proportion \\
\hline \multirow{4}{*}{$\begin{array}{c}\text { Number } \\
\text { of } \\
\text { computers }\end{array}$} & $1-5$ & 7 & $13.2 \%$ \\
\cline { 2 - 4 } & $6-10$ & 7 & $13.2 \%$ \\
\cline { 2 - 4 } & $11-20$ & 8 & $15.1 \%$ \\
\cline { 2 - 4 } & $21-50$ & 12 & $22.6 \%$ \\
\cline { 2 - 4 } & $101-100$ & 12 & $22.6 \%$ \\
\cline { 2 - 4 } & Over 201 & 4 & $7.5 \%$ \\
\hline
\end{tabular}


Table 3.Considerations analysis between PU,PEOU,EV

\begin{tabular}{|c|c|c|}
\hline Items & Crucial considerations & Votes \\
\hline $\begin{array}{c}\text { Perceived } \\
\text { usefulness } \\
(\text { PU) }\end{array}$ & Can be used anywhere & 19 \\
\cline { 2 - 3 } & Can be used in mobile devices & 18 \\
\hline $\begin{array}{c}\text { Perceived ease } \\
\text { of use } \\
\text { (PEOU) }\end{array}$ & $\begin{array}{c}\text { Reduce build and } \\
\text { maintenance costs }\end{array}$ & 25 \\
\cline { 2 - 3 } & $\begin{array}{c}\text { Pay fees according to the } \\
\text { usage }\end{array}$ & 19 \\
\hline $\begin{array}{c}\text { External } \\
\text { variables (EV) }\end{array}$ & $\begin{array}{c}\text { Improve employee work } \\
\text { efficiency }\end{array}$ & 16 \\
\hline
\end{tabular}

Table 4. Statistics of important factors of external variables

\begin{tabular}{|l|c|}
\hline \multicolumn{1}{|c|}{ Service items } & Scores \\
\hline $\begin{array}{l}\text { (1) Information and communication technology } \\
\text { application characteristics, and online sharing services }\end{array}$ & 7.4 \\
\hline (2) Office administration services & 6.4 \\
\hline (3) Customer relationship management (CRM) & 6.6 \\
\hline (4) Business operation and orders purchasing services & 6.7 \\
\hline (5) Operation financial management services & 6.4 \\
\hline (6) Human resource management services & 6.3 \\
\hline $\begin{array}{l}\text { (7) Business production and manufacturing } \\
\text { management services }\end{array}$ & 6.0 \\
\hline
\end{tabular}

\section{Conclusions and recommendations}

\subsection{Conclusion}

The relationship between perceived usefulness and perceived ease of use and behavior intention can be obtained based on the analysis conducted in this study. Perceived usefulness and perceived ease of use positively influence behavior intention, and also can verify and support the core structure of TAM [3,6,8]. Schepers and Wetzels[7] summarized a common conclusion that perceived usefulness as having a stronger impact on behavior intention than perceived ease of use. However, in this study,cloud servicesare network applications, and the conclusion that the influence of perceived ease of use is superior to perceived usefulness corresponds to the results obtained by King and $\mathrm{He}$ [9]. They posited that the perceived ease of use played a more important role in internet application. Although cloud systems possess advantages such as elasticity, multiplicity, the ability to extend, and availability on demand, the cost of implementing SaaS is still the foremost consideration for SMEs, meanwhile, basic advantages of SaaS systems, such as improving the work efficiency of employees, being used anywhere, and being used in mobile device, are also very vital.

\subsection{Contribution}

This study utilized TAM theory and questionnaires to understand the considerations of SMEs that use SaaS.Further, external characteristicvariables were introduced. Two behavior intentions of SMEs that employ SaaS were found to be most prominent in the study results.

\subsubsection{Usefulness after ease}

In the study, perceived ease of use not only had a greater impact on behavior intention than perceived usefulness, it also influences perceived usefulness positively. From these two points, a multiplier method in the cognitive stage is letting SMEs realize that SaaS systemscan have friendly interfaces, are easy to learn and use, and are not a waste of time because they have succinct interfaces with plain and brief illustrations. It can enhance their behavior intention and improve the possibility of actual actions.

\subsubsection{Intensifying system characteristics to enhance the intention of SMEs}

The results also indicate that system characteristics play a vital role in both perceived usefulness and perceived ease of use. Further, perceived usefulness can strengthen the information and communication technology applications in system characteristics, which are including online sharing service, business operation and orders purchasing services, and customer relationship management services, to satisfy the present and future demands of enterprises.

\subsubsection{Obtaining crucial value for future product development}

The results collected for cloud service providers, whether for product development in the future, marketing, or customer services, will be crucial references for vendors who can understand SaaS system functions of SMEs associated with this research.

\subsection{Research limitations and recommendations}

This study was ultimately restricted by time and resources, and there are three insufficient aspects that need further investigation: (1)Questionnaires should be designed according to business size and specific industry (such as information services, chain industry, and manufacturing), and external variables built. (2) Reliability and validity should be considered in the analysis to improve the accuracy of the results. (3) The effect of using SaaS for internet externalities should be discussed.

\section{References}


1. F. D. Davis, A technology acceptance model for empirically testing new end-user information systems: Theory and results. Massachusetts Institute of Technology,Sloan School of Management, (1986).

2. M. Fishbein,I. Ajzen, Belief, attitude, intention and behaviour:An introduction to theory and research,Boston,Massachusetts:Addison-Wesley (1975).

3. F. D. Davis, Perceived usefulness, perceived ease of use, and user acceptance of information technology. MIS Quart.,13(3), 319-340 (1989).

4. I. Ajzen, The theory of planned behavior. Organ.Behav.Hum.Decis.Process.,50(2), 179-211 (1991)

5. F. D. Davis, R. P. Bagozzi, P. R. Warshaw, User acceptance of computer technology: A comparison of two theoretical models. Manag. Sci.'982-1003 (1989).

6. V. Venkatesh, F. D. Davis, A theoretical extension of the technology acceptance model: Four longitudinal field studies. Manag.Sci., 46(2), 186-204 (2000).

7. J. Schepers, M. Wetzels, A meta-analysis of the technology acceptance model: Investigating subjective norm and moderation effects. Info. \&Manag., 44(1), 90-103 (2007).

8. V. Venkatesh, H.Bala, Technology acceptance model 3 and a research agenda on interventions. Decis. Sci., 39(2), 273-315 (2008).
9. W. R. King,J. He, A meta-analysis of the technology acceptance model. Info.\&Manag.,43(6), 740-755 (2006).

10. V. Venkatesh, M. G. Morris, Why don't men ever stop to ask for directions? Gender,social influence, and their role in technology acceptance and usage behavior. MIS Quart., 24(1), 115-139 (2000).

11. V. Venkatesh, Determinants of perceived ease of use: Integrating control, intrinsic motivation, and emotion in the technology acceptance model. Info.Syst.Res., 11(4), 342-365 (2000).

12. V. Venkatesh, M. G. Morris, G.B. Davis, F. D. Davis, User acceptance of information technology: Toward a unified view. MIS Quart., 27(3), 425-478 (2003).

13. T. J. Larsen, A. M. Sorebo, O. Sorebo, The role of task-technology fit as usersmotivation to continue information system use. Comput.Hum.Behav.25(3), 778-784(2009).

14. J. M. O. Egea, M. V. R. Gonzales, Explaining physicians'acceptance of EHCR systems: An extension of TAM with trust and risk factors. Comput.Hum.Behav.,27(1),319-332 (2011).

15. T. S. Behrend, E. N. Wiebe, J. E. London, E. C. Johnson, Cloud computing adoption and usage in community colleges. Behav.\&Info.Tech., 30(2), 231240

(2011). 